\title{
Cement Salvage of Instrumentation-Associated Vertebral Fractures
}

\author{
R. Xu, K. O'Connor, G. Krol, Y. Yamada, M. Bilsky, I. Laufer, and E. Lis
}

\begin{abstract}
BACKGROUND AND PURPOSE: Spinal instrumentation plays a key role in the treatment of spinal instability in patients with metastatic tumors. Poor bone quality, radiation, and diffuse osseous tumor involvement present significant challenges to spinal stabilization with instrumentation and occasionally result in postinstrumentation compression fractures. Vertebral cement augmentation has been effective in the treatment of painful tumor-related compression fractures. Our objective was to describe cement augmentation options in the treatment of vertebral compression fractures associated with spinal instrumentation in patients with metastatic tumors.
\end{abstract}

MATERIALS AND METHODS: Patients who underwent percutaneous vertebral cement augmentation in the treatment of instrumentation-associated vertebral compression fractures between 2005 and 2011 were included in the analysis. Only fractures that occurred within the construct or at an adjacent level were included. The change in Visual Analog Scale and need for further surgery were analyzed.

RESULTS: Eleven patients met the inclusion criteria, with 8 tumors located in the thoracic spine and 3 tumors in the lumbar spine. The median time between instrumented surgery and vertebral augmentation was 5 months (1-48 months) and the median follow-up after cement augmentation was 24 months (4-59 months). A total of 22 vertebrae that were either within or immediately adjacent to the surgical instrumentation underwent vertebral augmentation. All patients reported a decrease in their pain scores (mean decrease: 6 Visual Analog Scale points; $P<.003$ ). One patient required reoperation after cement augmentation. None of the patients experienced vertebral cement augmentation-related complications.

CONCLUSIONS: Vertebral cement augmentation represents a safe and effective treatment option in patients with recurrent or progressive back pain and instrumentation-associated vertebral compression fractures.

ABBREVIATIONS: VAS $=$ Visual Analog Scale; SRS $=$ stereotactic radiosurgery

$\mathrm{T}$

he role of surgery in the treatment of metastatic spinal tumors has been firmly established as an effective and safe method for spinal cord decompression and stabilization of the spine. The goals of surgery for spinal metastases remain palliative and include preservation or restoration of neurologic function and pain control. Tumor control is largely accomplished using radiation and chemotherapy. In patients with metastatic spinal tumors, spi-

Received September 9, 2013; accepted after revision April 2, 2014.

From the Departments of Neurosurgery (R.X., K.O., M.B., I.L.), Spine Tumor Center (M.B., I.L., E.L.), Radiology (G.K., E.L.), and Radiation Oncology (Y.Y.), Memorial Sloan-Kettering Cancer Center, New York, New York; Department of Neurological Surgery (M.B., I.L.), Weill Cornell Medical College, New York-Presbyterian Hospital, New York, New York; Mount Sinai School of Medicine (K.O.), New York, New York; and Department of Medical Biophysics (R.X.), Institute of Physiology and Pathophysiology, Heidelberg University, Heidelberg, Germany.

Please address correspondence to Eric Lis, MD, Department of Radiology, Memorial Sloan-Kettering Cancer Center, 1275 York Ave, New York, NY 10021; email: lise@mskcc.org

三 Indicates article with supplemental on-line table.

http://dx.doi.org/10.3174/ajnr.A4037 nal instrumentation is required in most cases to provide spinal stability after circumferential spinal cord decompression. Spinal fixation in this patient population can be quite challenging because of extensive osteoporosis and lytic tumor destruction. Furthermore, chest wall resection may be required, further destabilizing the spine and increasing the risk of fixation failure. Prior spine radiation results in increased risk of vertebral compression fractures. ${ }^{1-3}$ Failure of fixation may require interruption or delay of systemic or radiation therapy, increasing the risk of local or systemic tumor progression. Vertebral compression fractures either within or adjacent to the surgical construct often result in either recurrent or progressive back pain.

Percutaneous vertebral cement augmentation (ie, balloon kyphoplasty/vertebroplasty) has been established as a safe and effective method of quickly achieving pain control in osteoporotic and tumor-related compression fractures. ${ }^{4,5}$ Cement has also been used to reinforce screws at the time of insertion. ${ }^{6,7}$ However, little information exists regarding its use as a salvage technique for 
instrumented patients who develop recurrent back pain secondary to new vertebral compression fractures within or adjacent to their surgical construct. We report a series of patients in whom percutaneous vertebral cement augmentation was used as an initial treatment of symptomatic instrumentation or junctional fractures in place of open hardware revision.

\section{MATERIALS AND METHODS \\ Patient Population}

Patients who underwent kyphoplasty or vertebroplasty and surgery for the treatment of spinal metastatic tumors between 2005 and 2011 were included in the study. A waiver of institutional review board authorization and informed consent was obtained from the institution to collect the existing data regarding these patients. Among the 29 patients who fit these inclusion criteria, 18 patients were excluded because the postcement augmentation follow-up was less than 2 months, they underwent cement augmentation before surgical stabilization, or the cement augmentation levels were more than 1 level outside of the instrumented levels. The charts and imaging studies of the remaining 11 patients were retrospectively reviewed for tumor histology, tumor level, decompression, instrumentation and cement augmentation levels, further revision surgery, and Visual Analog Scale (VAS) scores.

\section{Surgery}

All patients underwent separation surgery, an open surgical technique that separates epidural disease to reconstitute the thecal sac and posterior stabilization, followed by postoperative radiation therapy. ${ }^{8}$ To provide circumferential thecal sac decompression at the level of epidural extension of the tumor, laminectomy with bilateral or unilateral facetectomy and resection of the ventral epidural tumor with very limited vertebrectomy were performed. Spinal stabilization was provided by posterolateral fixation at least 2 levels above and below the tumor. All patients were treated with adjuvant radiation therapy that consisted of either conventional external-beam radiation or stereotactic radiosurgery (SRS) that was selected based on tumor histology and prior radiation history.

\section{Cement Augmentation}

All patients had cross-sectional imaging of the spine before the procedure usually consisting of MR imaging and often a CT scan. This determined which vertebrae to augment and helped in the planning of the trajectory of the introducer needles for subsequent cement augmentation. The procedure was performed under general anesthesia in the interventional radiology suite that has both fluoroscopic and CT (conebeam as well as collimated) capabilities. Both CT and fluoroscopy were used for placement of the introducer needles. The trajectory of the introducer needles was dictated by the hardware and anatomy (Fig 1). During the fluoroscopic portion of the procedure, oblique views of the spine were often required in addition to the more standard anterior-posterior and lateral views to "throw off" the hardware and to allow better visualization of the introducer needles and cement infusion. If there was any question regarding needle or cement location, an intraprocedural conebeam CT scan was obtained. Deciding between balloon kyphoplasty and vertebroplasty was determined during the procedure by needle trajectory and the anatomy. At the levels without intrapedicular screws or with only a unilateral

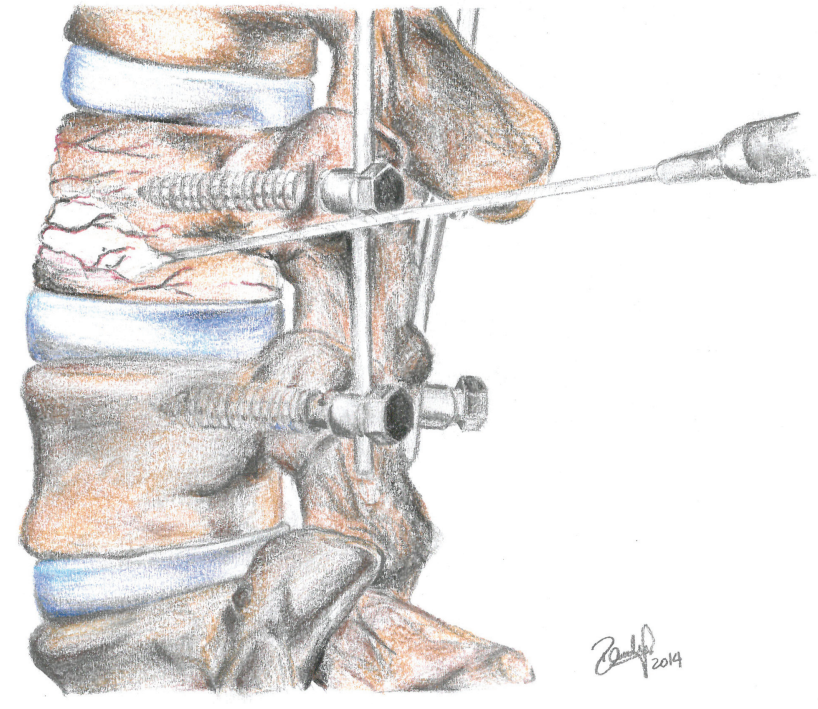

FIG 1. Percutaneous vertebral cement augmentation for an instrumentation-associated vertebral fracture. Schematic showing the placement of the introducer needle and cement infusion, the trajectory of the kyphoplasty needle being dictated by the presence of existing hardware, and anatomic structures.

screw, an inflatable bone tamp (Medtronic MIS, Sunnyvale, California) was used before the cement infusion (kyphoplasty). At levels with previously placed bilateral screws, the trajectory of the introducer needle was extrapedicular, often at the superior or inferior extremes of the vertebral body thereby obviating the ability to place a bone tamp. In these cases vertebroplasty was performed, usually through a curved AVAflex needle (Carefusion, Waukegan, Illinois). The use of the curved needle was particularly helpful in directing the cement into different regions of the vertebral body when surgical screws limited the position of the introducer needle.

The cement was hand injected coaxially through the introducer needle under fluoroscopic visualization, with repeat CT imaging performed if there was a question of extravasation into the spinal canal or neural foramina. The cement used was either the standard high-viscosity radiopaque polymethylmethacrylate or Cortoss bone augmentation material (Stryker Neurovascular, Fremont, California), which is a nonresorbable composite. The latter has an advantage that a small amount can be mixed on demand, which is particularly helpful if switching back and forth between CT and fluoroscopy is required.

\section{Data Analysis}

Statistical analysis was performed using SPSS 20.0 (IBM, Armonk, New York). A Wilcoxon signed ranks test was used to compare the prekyphoplasty and postkyphoplasty VAS scores.

\section{RESULTS}

Individual patient and treatment information are summarized in the On-line Table. The median age at time of postsurgical cement augmentation was 60 years (range: $38-71$ years). Median follow-up after cement augmentation was 24 months (range: 4-59 months), and the median time between instrumentation and salvage cement augmentation was 5 months (range: $1-48$ months). Eight tumors were located in the thoracic spine and the remaining 3 were located in the lumbar spine. All patients underwent cement 

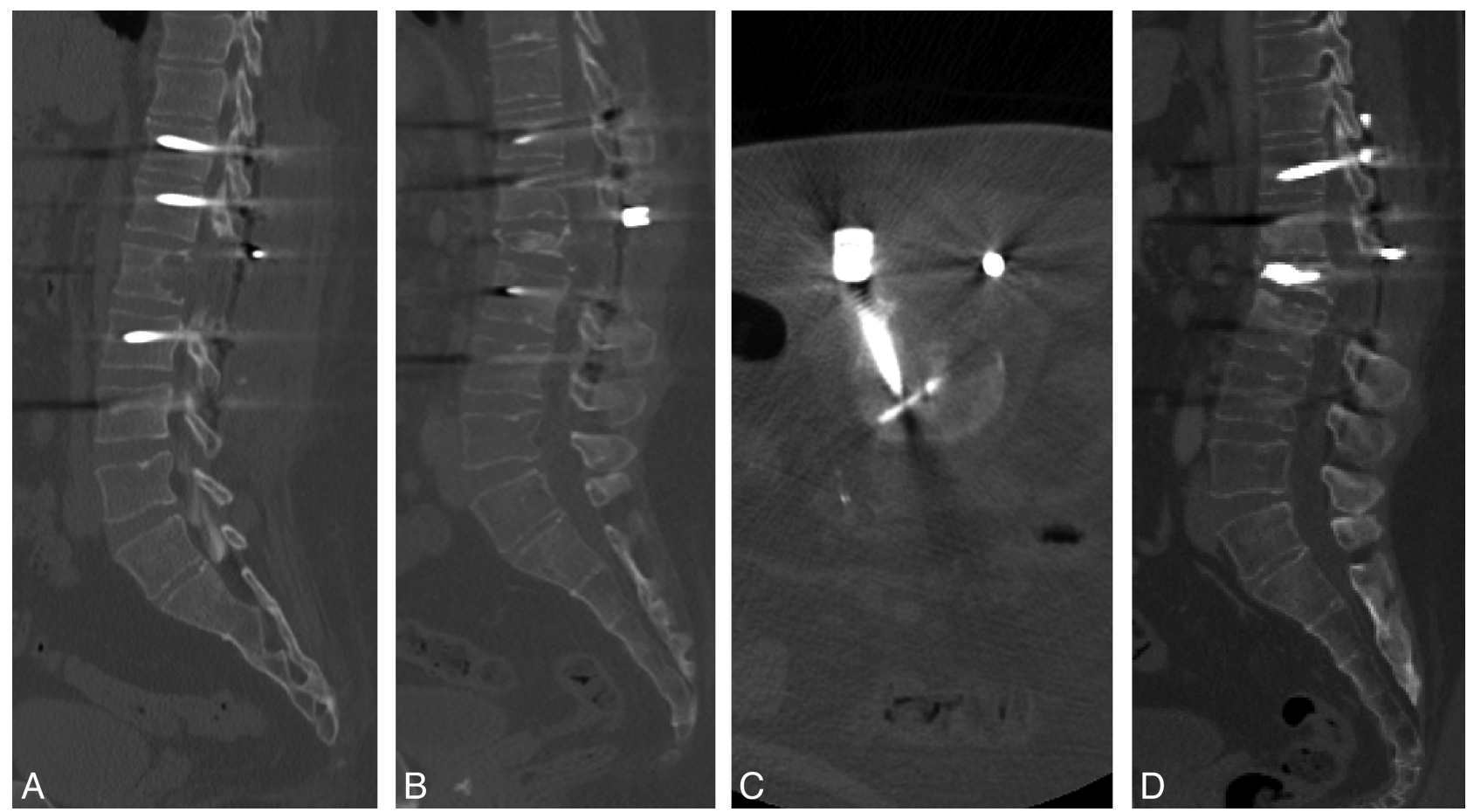

FIG 2. A 38-year-old man with renal cancer with L1 metastasis treated with surgical decompression followed by SRS. A, Reconstructed sagittal CT of the lumbar spine obtained post L1 laminectomy. B, Follow-up CT scan 23 months later showing a moderate L1 collapse. C, Intraprocedural CT confirming an adequate trajectory through the L1 vertebral body. D, Postaugmentation CT scan showing good filling of the L1 vertebral body.

augmentation after developing new painful compression fractures. The pain rather than the radiographic finding was the indication for intervention. Eight of the cement augmentation procedures were done at the levels of the top or bottom screws or immediately adjacent to these levels. The remaining 3 patients had cement augmentation in the middle of the construct.

The mean prekyphoplasty VAS score was 8.4 (range 4-10) and postkyphoplasty score was 1.5 (range $0-5$ ). All patients reported a decrease in their pain scores. The mean decrease in the VAS score was 6 points $(P<.003)$.

One patient required surgery after kyphoplasty. The patient initially underwent L1 decompression and T11-L3 stabilization for a renal cell metastasis (Fig 2A). One year after the initial operation, the patient developed severe back pain and was found to have a new L1 compression fracture; however, the patient's hardware appeared intact (Fig 2B). The patient underwent a L1 kyphoplasty with significant pain relief, from 10/10 to $1 / 10$ (Fig 2C, $-D)$. Five months after the kyphoplasty, the patient developed new back pain and x-rays revealed a unilateral rod fracture. The rod was replaced and the back pain resolved.

None of the patients experienced vertebral cement augmentation-related complications such as neural element compression or cement embolization.

\section{Case Example}

A 62-year-old man with metastatic melanoma underwent singlefraction SRS ( $24 \mathrm{~Gy}$ ) to L4. Three months after radiation, he developed an L4 burst fracture and mechanical radiculopathy requiring an L3-L5 posterolateral instrumentation and fusion with left L4-L5 facetectomy. One year later, the patient developed an L2 metastasis. Initially he underwent an L2 kyphoplasty; however, because of the progression of radicular pain and posterior element instability he required extension of instrumentation to T12 and right-sided transpedicular decompression of the epidural tumor. Four years after the initial surgery, the patient developed recurrence of back pain and was noted to have a compression fracture at L4 and endplate infractions at L3 and L5 without evidence of tumor progression (Fig 3A). This patient underwent cement augmentation at L3-L5, resulting in significant decrease of pain symptoms (VAS $10 / 10$ to 2/10). At L4, kyphoplasty was performed; the needle was advanced into the vertebral body via the left pedicle, and an inflatable bone tamp was used before cement infusion (Fig 3B, $-C$ ). At levels L3 and L5, where bilateral screws were present, the trajectory of the introducer needles was extrapedicular, therefore obviating the ability to place a bone tamp; instead, vertebroplasty was performed through a curved AVAflex needle. At L3, a lateral parapedicular approach was undertaken. At L5 the guide needle was inserted via a superior, extrapedicular approach and the augmentation needle was then advanced coaxially, allowing access to both the contralateral and unilateral side (Fig $3 B,-C$ ).

\section{DISCUSSION}

The treatment of spinal metastases is performed with the palliative goals of preservation or restoration of neurologic function and spinal stability, pain control, and local tumor control. Surgery is indicated for patients with metastatic spinal tumors in the setting of spinal cord compression and spinal instability. ${ }^{9}$ Instrumentation restores spinal stability after circumferential decompression and osseous infiltration by tumor. Generally patients with metastatic tumors require systemic therapy, which requires

AJNR Am J Neuroradiol 35:2197-201 Nov 2014 www.ajnr.org 

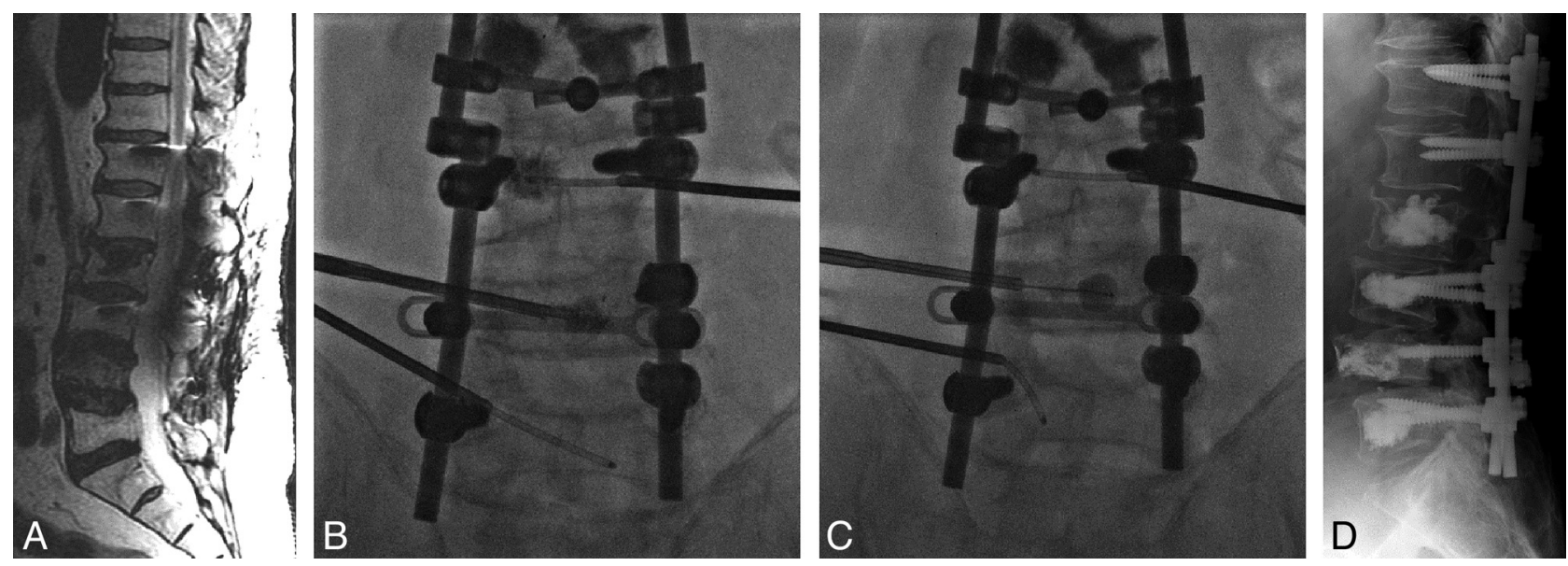

FIG 3. A 62-year-old man status post prior L2 kyphoplasty and radiation therapy for melanoma metastasis with subsequent recurrence and surgical decompression. $A$, The patient developed a partial collapse deformity of $L 4$ with early endplate infractions $L 3$ and $L 5$ as demonstrated on MR imaging. $B$, Anterior-posterior fluoroscopic image showing an inflated bone tamp in L3, with curved needles in L3 and L5. C, Anteriorposterior image a few moments later showing cement infusion into L3 and L4. Notice the repositioning of the curved needle in L5. D, Postaugmentation sagittal $\mathrm{x}$-ray demonstrating good filling of vertebral bodies.

coordination with surgery and radiation. Spinal radiation is generally administered 3-4 weeks after surgery to decrease the risk of wound dehiscence or infection. Systemic therapy is administered after radiation and is of paramount importance in preventing systemic progression of cancer. Postoperative wound complications or hardware failure may require significant delay in chemotherapy administration and may have disastrous implications, with systemic progression leading to the demise of the patient or requiring additional surgery and radiation.

While the beneficial role of surgery has been thoroughly documented in the treatment of patients with spinal metastases, these operations may be associated with a wide range of potential complications. The reported perioperative complication rate range is $19 \%-50 \% .{ }^{10-12}$ The hardware failure rate has been reported to be $2.2 \%-16 \%$. Hardware-related complications include dislodgement of titanium cage, screw, hook, rod, or plate back-out, or breakage and adjacent level fractures. Generally, symptomatic hardware malfunction compromises spinal stability and requires patients to return to the operating room to replace the fractured components and often to extend the fixation to adjacent levels. In oncologic patients, multilevel tumor infiltration along with chest wall involvement further complicates the stabilization, as does underlying poor bone quality often secondary to osteoporosis and prior radiation. Avoidance of multiple hardware revisions is crucial in continuation of chemotherapy and radiation and in avoidance of high-risk reoperations.

Percutaneous vertebral cement augmentation has been established as an effective treatment for painful fractures in patients with metastatic spinal tumors. ${ }^{4}$ The Spine Oncology Study Group $^{13}$ made a strong recommendation for the use of vertebral cement augmentation in patients with symptomatic osteolytic metastases and compression fractures. The group conducted a systematic review of the literature that confirmed that kyphoplasty or vertebroplasty consistently relieves mechanical axial pain and improves functional status. Furthermore, the investigators of the Cancer Fracture Evaluation study ${ }^{5}$ randomized 134 patients with painful compression fractures to undergo kyphop- lasty or nonsurgical management and found a significant improvement in the pain and function in the treatment group at 1 -month follow-up. Complications were very rare, with 1 patient experiencing anesthesia-related non-Q-wave myocardial infarction and 1 patient developing an adjacent-level vertebral body fracture 1 day after the kyphoplasty. Thus, vertebral cement augmentation provides a safe and effective minimally invasive treatment option for cancer-related vertebral fractures that can be performed on an outpatient basis.

While the procedure of postinstrumentation vertebral body cement augmentation is similar to standard percutaneous vertebral cement augmentation procedures performed in nonsurgically stabilized patients, there are some unique technical challenges. The fixation hardware often consists of bilateral intrapedicular screws and posterior stabilization rods, which alters access to the vertebral body. The presence of pedicle screws essentially eliminates the transpedicular approach to the vertebral body. The screws within the vertebral body are typically lateral, making access to the central aspect of the vertebral body challenging. Fluoroscopic imaging is more difficult because the introducer needle tips may be obscured or silhouetted by the surgical hardware. In the lateral plane under fluoroscopy, the presence of intrapedicular screws will also obscure a portion of the spinal canal and ventral epidural space. This is of critical importance during the infusion of the cement as the posterior extent of the cement may not be readily apparent. If the vertebral body to be augmented is at the level of tumor resection, often the standard fluoroscopic imaging landmarks are absent. The pedicles and posterior elements typically have been resected and or previously destroyed by tumor. This not only makes fluoroscopic access challenging, but also the absence of the posterior elements removes the bony protection of the thecal sac. Similarly, if the costovertebral junction has been removed, the thorax is more vulnerable to penetration by the introducer needles. Multiple oblique fluoroscopic trajectories may be required to optimize the visualization of the needle trajectory and cement. If needle placement 
trajectory or the posterior extent of cement is not clear with fluoroscopy, an intraprocedural conebeam CT can be obtained.

In addition to the hardware and postsurgical osseous changes, postsurgical soft tissue changes need to be considered. In particular, the presence of a paraspinal fluid collection, seroma, or pseudomeningocele will need to be avoided and require modification of the trajectory of the introducer needle. This reinforces the necessity of having a preprocedural cross-sectional imaging study to help determine the best trajectory of the augmentation needle into the collapsed vertebrae.

The current patient series documents the feasibility of percutaneous vertebral cement augmentation in the treatment of symptomatic vertebral compression fractures within or immediately adjacent to pedicle fixation constructs. The data show that cement reinforcement provides effective pain relief in instances of junctional fractures as well as fractures within the construct. Thus, in place of open hardware revision and extension, patients undergo an outpatient procedure with minimal risk of morbidity. Our report includes the results of a small series of consecutive patients who were treated with this technique, and a larger prospective cohort will be necessary to determine the optimal candidates for this treatment and to provide more generalizable outcome data. Furthermore, in some patients, the position of the instrumentation may prohibit safe cement augmentation. Cement salvage of hardware-related fractures provides a safe and well-tolerated alternative to open surgery that does not require interruption of systemic therapy.

\section{CONCLUSIONS}

Surgery for metastatic spinal cancer is a palliative measure. Poor quality of bone and tumor progression can lead to new symptomatic compression fractures. The use of percutaneous vertebral cement augmentation in these situations can be extremely beneficial for the patient by effectively relieving pain with an outpatient procedure that does not require interruption of systemic therapy or radiation.

Disclosures: Yoshiya Yamada_UNRELATED: Consultancy: Varian Medical Systems, Speakers Bureau for the Institute for Medical Education, Comments: CME-approved lectures; Board Membership: American Brachytherapy Society; Travel/Accommodations/Meeting Expenses Unrelated to Activities Listed: American Brachytherapy Society; Grants/Grants Pending: Medtronics, ${ }^{\star}$ Comments: for an image-guided brachytherapy study. Mark Bilsky—UNRELATED: Consultancy: SpineWave and
DePuy Synthes. Ilya Laufer-UNRELATED: Consultancy: SpineWave and DePuy Synthes. Eric Lis-UNRELATED: Consultancy: Medtronic; Payment for Lectures (including service on speakers' bureaus): Medtronic. *Money paid to the institution.

\section{REFERENCES}

1. Boehling NS, Grosshans DR, Allen PK, et al. Vertebral compression fracture risk after stereotactic body radiotherapy for spinal metastases. J Neurosurg Spine 2012;16:379-86

2. Cunha MV, Al-Omair A, Atenafu EG, et al. Vertebral compression fracture (VCF) after spine stereotactic body radiation therapy (SBRT): analysis of predictive factors. Int J Radiat Oncol Biol Phys 2012;84:e343-49

3. Rose PS, Laufer I, Boland PJ, et al. Risk of fracture after single fraction image-guided intensity-modulated radiation therapy to spinal metastases. J Clin Oncol 2009;27:5075-79

4. Fourney DR, Schomer DF, Nader R, et al. Percutaneous vertebroplasty and kyphoplasty for painful vertebral body fractures in cancer patients. J Neurosurg 2003;98:21-30

5. Berenson J, Pflugmacher R, Jarzem P, et al. Balloon kyphoplasty versus non-surgical fracture management for treatment of painful vertebral body compression fractures in patients with cancer: a multicentre, randomised controlled trial. Lancet Oncol 2011;12: 225-35

6. Amendola L, Gasbarrini A, Fosco M, et al. Fenestrated pedicle screws for cement-augmented purchase in patients with bone softening: a review of 21 cases. J Orthop Traumatol 2011;12:193-99

7. Sawakami K, Yamazaki A, Ishikawa S, et al. Polymethylmethacrylate augmentation of pedicle screws increases the initial fixation in osteoporotic spine patients. J Spinal Disord Tech 2012;25:E28-35

8. Laufer I, Iorgulescu JB, Chapman T, et al. Local disease control for spinal metastases following "separation surgery" and adjuvant hypofractionated or high-dose single-fraction stereotactic radiosurgery: outcome analysis in 186 patients. J Neurosurg Spine 2013;18:207-14

9. Patchell RA, Tibbs PA, Regine WF, et al. Direct decompressive surgical resection in the treatment of spinal cord compression caused by metastatic cancer: a randomised trial. Lancet 2005;366:643-48

10. Falicov A, Fisher CG, Sparkes J, et al. Impact of surgical intervention on quality of life in patients with spinal metastases. Spine 2006; 31:2849-56

11. Finkelstein JA, Zaveri G, Wai E, et al. A population-based study of surgery for spinal metastases. Survival rates and complications. J Bone Joint Surg Br 2003;85:1045-50

12. Wise JJ, Fischgrund JS, Herkowitz HN, et al. Complication, survival rates, and risk factors of surgery for metastatic disease of the spine. Spine 1999;24:1943-51

13. Mendel E, Bourekas E, Gerszten P, et al. Percutaneous techniques in the treatment of spine tumors: what are the diagnostic and therapeutic indications and outcomes? Spine 2009;34:S93-100 